\title{
A systematic review protocol to explore the prevalence and impacts of neurodevelopmental disorders in the care experienced by the looked after population
}

Nicola Jane Heady ( $\sim 886990 @ s w a n s e a . a c . u k$ )

Swansea University https://orcid.org/0000-0002-6689-0494

Alan Watkins

Swansea University

Ann John

Swansea University

Hayley Hutchings

Swansea University

\section{Protocol}

Keywords: Looked after child, Prevalence, Mental, Neurodevelopmental, Developmental, Disorder, Systematic review

Posted Date: April 2nd, 2020

DOI: https://doi.org/10.21203/rs.3.rs-19684/v1

License: (c) (i) This work is licensed under a Creative Commons Attribution 4.0 International License.

Read Full License 


\section{Abstract}

Background: A looked after child (LAC) is defined as a child or young adult, that has been placed in either a foster care, kinship, residential or adoption care setting. While embedded in the care of these services, these children still develop debilitating disorders that significantly impact their future overall wellbeing. Prevalence of these disorders are often depicted under broad, umbrella categories such as mental, behavioural or developmental disorders. What specific disorders fall under these broad categories is limited in research. This review aims to explore the prevalence and early impacts of neurodevelopmental disorders in the LAC and if feasible, compare prevalence with those children who are not looked after (NoN-LAC).

Disorders such as dyslexia, bipolar and autism which are classified as neurodevelopmental or associated disorders in the expert field of genetics and neuropsychiatry will be explored. These disorders significantly impact on emotions, memory, ability to learn and socialize and are diagnosed solely based on behaviour. As a result; attaining a diagnosis can take years due to the associated complex behavioural symptomology.

With an increasing rate of children entering the care system on a global level; it is an appropriate time to examine the prevalence and impact of these neurodevelopmental disorders in this already, vulnerable population.

Methods: A literature search will be conducted using several multiple databases such as PubMed, PsychINFO and Web of Science Core Collection. Secondary literature, grey literature and government publications will also be searched systematically to further identify any eligible studies. Children involved in foster, residential, adopted or kinship care will be identified as a LAC. NoN-LAC will be children who are not placed in the looked after care setting. The age limit is 25 years as the review aims to explore the earlier impacts these disorders may have on the overall wellbeing of these children. Appropriate statistical techniques will be utilised and adapted throughout the review dependent on the research designs of the eligible studies.

Discussion: This review could identify gaps in LAC research and potentially contribute to the understanding of the adverse life outcomes that many of these children are predicted to attain.

Systematic review registration: PROSPERO Registration number: CRD4201913103

\section{Background}

Research continues to depict a bleak future for looked after children; predicting inauspicious health and social outcomes [1-8]. Although, research in LAC is still limited; poor outcomes associated with mental health, disability, suicide, criminal system involvement, teenage parenthood, substance misuse and educational attainment have become the predicted 'norm' for these children [1-8]. These discouraging findings have prompted governments to address these concerns; positioning this population at the 
forefront of policy and legislation $[9,10]$. Yet despite being classified as a high-risk group; factors such as multiple placements, residential moves, poor screening in development and education, delays in service provision, limited data collation, consent and segregated service sectors are still suggested to contribute to these outcomes [11-13].

Situated within these poor outcomes and currently, major priority objectives for government; are the areas of mental health and educational attainment [9]. Both factors are suggested to be intrinsically linked; forming and underpinning the fundamental building blocks to achieving subjective wellbeing and increasing social mobility [15-16]. Although major priorities for all children in the UK; research proposes that LAC are nine times more likely to have a special educational need due to social, emotional, behavioural, developmental and learning difficulties and four times more likely to have a mental health disorder compared to those not in the care setting and [14, 17-19].

The prevalence of psychiatric disorders is well documented in research as being higher in the LAC population; often suggested to be as a result of adverse childhood experiences or poor socio-economic environments [20 - 24]. However, these prevalence studies are often depicted and categorised under broad headings; such as mental, behavioural, emotional or developmental disorders [18, 21, 25]. Although, significant findings; limited within secondary literature is a more in-depth, individual analysis of what specific disorders represent these broad categories.

All psychiatric disorders can significantly impact on the individuals' mental health, emotions, memory, ability to learn and socialize at some point in their life [26, 27]. Some may affect the individual on an intermittent basis or manifest during periods of immense stressful or challenging life events [26, 27]. However; there is emerging research in the psychiatric and neurological field that suggest that some disorders originate during or after birth and are classified as 'neurodevelopmental' disorders or are associated as such by leading experts in the field [28-32]. These disorders, like other psychiatric disorders significantly cause developmental deficits in emotional regulation, memory, and ability to learn and socialize.These disorders however remain for life [28-32].

There is a wide spectrum of neurodevelopmental disorders which can be diagnosed with a medical test; disorders such as Prader-Willi Syndrome or Fragile X Syndrome [33, 34]. However, many neurodevelopmental disorders can only be diagnosed solely based on behaviour [35, 36]. As a result, due to the often similar and complex behavioural symptomology associated with these neurodevelopmental disorders; many children do not attain a diagnosis until they reach adolescent or adult age [36 - 38]. Furthermore, emerging research proposes that many of these neurodevelopmental disorders co-exist and therefore, make diagnosis even more challenging for the professional [39]. This lack of early diagnosis and intervention can leave the child struggling in the home, school and social setting with no support or understanding; attempting to navigate a world that makes no sense to them which frequently results in a detrimental impact on their health and social wellbeing [36-38, 40].

This review will explore the prevalence and impact of neurodevelopmental disorders that are based on classifications defined in previous studies in the field of neurodevelopmental disorders but cannot be 
medically tested for [28-32]. The disorders of interest for this review will be Foetal Alcohol Spectrum Disorder, Reactive Attachment Disorder, Attention Deficit Hyper Disorder (ADHD), Bipolar, Schizophrenia, Obsessive Compulsive Disorder, Eating Disorders, Autism Spectrum Disorder (Pervasive Development Disorder, Asperger), Mathematics Disorder (Dyscalculia), Intellectual Disability, Reading and written Disorder (Dyslexia), Speech and Language Impairment, Social (Pragmatic) Communication Disorder, Tic Disorder, Stereotypic Movement Disorder (Dyspraxia) [41].

Historically, they have often been omitted or not elaborated upon in research for varying reasons; such as low sample numbers $[1,3,20]$. Nevertheless, with increased awareness of the diversity of how these disorders manifest in the child increasing in the psychiatric field and diagnostic accuracy improving; research into these neurodevelopmental disorders has much improved [34-36]. Willis et al (2017) recently conducted a systematic review and found that the prevalence of Attention Deficit Hyperactivity Disorder (ADHD) was suggested to be higher in in the LAC population compared to NoN-LAC [42]. Although, a significant finding, this review differs as the inclusion criteria includes two further 'looked after' groups; children who have been adopted or children who have been placed in a kinship care setting. Additionally, this review will screen both published and unpublished articles to alleviate anticipated publication bias due to the nature of the outcomes of interest $[42,43]$.

Nevertheless, what is integral and of significant importance is that early identification and intervention of these neurodevelopmental disorders are key determinants for improving overall future health and social outcomes $[44,45]$. For the front-line professional who is primarily responsible for referring the LAC to appropriate services; research ascertains that many professionals still address this area as challenging, creating potentially significant barriers for early intervention [46]. Difficult to diagnose and often concealed from front line professionals, they are often inadvertently overlooked by the professional; as the urgency to treat or provide the child with support overrides further exploration of the underlying behavioural symptomology $[47,48]$. This can often lead to neurodevelopmental disorders being misdiagnosed or missed altogether in the general population and LAC population [47, 48]. Unfortunately, as a result, these underlying neurodevelopmental disorders do not become apparent until the child reaches a mental health crisis point; particularly the adolescent era where services are then forced to intervene $[47,48]$.

Furthermore, the results attained from this review might add some value to the existing body of literature that suggest that there might be familial risk, heritability and possible genetic factors associated with some of these disorders [28-32, 49]. From an economic and policy perspective; with rising statistics of children entering the care system and an urgent need to meet their needs; this review proposes it is an appropriate time to add to the existing literature in this field. The review will aim to address the following research questions:

1. How does the prevalence of these neurodevelopmental disorders in the Looked after population compare with those children who are NoN-LAC? 
2. What early impact do these disorders have on the health and social wellbeing of the Looked after child?

\section{Methods}

This protocol has been registered with the PROSPERO database for systematic reviews (Registration number: CRD4201913103) and will be reported in accordance with the guidelines outlined in the Preferred Reporting items for Systematic Reviews and Meta-Analyses Protocols (PRISMA-P). The review will follow the PRISMA-P guidance and all processes will be clearly defined in the review [50].

\section{Search Strategy}

The PICO approach has been used to help define the primary research question and formulate the search strategy.

Does the child $(P)$ who is in a looked after care setting $(I)$ compared to those children that are not placed in the care setting $(C)$ have a higher prevalence $(0)$ of these neurodevelopmental disorders?

As observational and descriptive studies draw inference from a sample to a population; where there are limits in logistics and ethical considerations; it is anticipated that these studies will be the most prevalent in this review. However, all designs will be included providing they meet the inclusion and quality criteria [51].

The following databases will be searched to identify relevant primary literature for the review: PubMed, ASSIA, IBSS, Web of Science, PsychINFO, Scopus, Psych articles, Social Care Online. The proposed databases were selected based on those identified in other peer reviewed studies that explored similar outcomes of interest and were agreed and deemed appropriate by all authors [54,55].

The review will additionally systematically hand search published and unpublished secondary, grey and governmental literature as they can be a rich information source for exploring citations and reference lists to further identify any new primary articles. Furthermore, exploring unpublished literature will contribute to alleviating potential publication bias $[43,53]$.

Due to the breadth of the research questions; there will be two stages to the search strategy. The first stage will explore the prevalence of these neurodevelopmental disorders in the LAC population and if feasible compare to NoN-LAC. The second stage will explore any impacts on health and social wellbeing as a result of having these disorders.

\section{Eligibility Criteria}

No restriction will be placed on the age of publication. Publications in the English language and international literature will be included; however, it is recognised that there are limitations to this approach. 
The legal definition for a 'Looked After Child' varies on an international level, however the review will be guided by the legal definitions underpinning the United Kingdom (UK), derived from both the Children Act 1989 and the Children Act 1995, Scotland (RCPCH, 2015). There will be a broad restriction on the terminology used to identify a child in care (adopted, residential, LAC, adopted, out of home care, foster child, kinship care) to ensure the review encapsulates the majority of children who are or have been in care; which coincides with governmental legislation, policy and third sector terminology for the definition of this population (RCPCH, 2015).

There will be no restriction on the care setting or time placed in a care as many of these children transition into different care settings such as adoption; residential or group care; reside with close family members or re-enter the care system [56]. The only restriction is that the child will have been placed in a care setting for over 24 hours which coincides with UK legislation. NoN-LAC will be defined as children or young adults that are not in a care setting or have never been in the care setting. There are limitations to this, as a child may have re-entered the care setting pre or prior to when the study collated the data [56]. However, this will be acknowledged as a limitation in the review.

As there is a duty of care to support some of these children up to the age of 25 in the UK; this will be the upper limit to the restriction. This will enable the review to capture those individuals who might have been diagnosed later in their life with these neurodevelopmental disorders. Additionally, this time period will enable the review to explore the earlier impacts on the health and social wellbeing of these children, as a result of having these neurodevelopmental disorders; for earlier intervention purposes. Additional adverse life experiences after leaving the care setting are often associated with this population; therefore, going any higher in age although a significant knowledge gap in research; could confound the results of the review [56].

Synonyms associated with the neurodevelopmental disorders being explored as detailed in Table 1 will be included in the search strategy. As these neurodevelopmental disorders are life-long; this review aims to attain a life-time prevalence and compare prevalence between the LAC population and their NoN-LAC peers [31]. Only comparative studies that explore prevalence of these neurodevelopmental disorders between these two groups will be used to conduct a meta-analysis. With the introduction of the new International Classification of Diseases 11th Revision (ICD-11) guidelines which acknowledge that many of these neurodevelopmental disorders now co-exist; this review will include studies that have prevalence rates for children who have more than one diagnosis [57].

Only studies that have confirmed diagnoses, or have used diagnostic codes defined by the diagnostic statistical manuals such as the Diagnostic and Statistical Manual of Mental Disorders (DSM) and International Classification of Diseases (ICD) or standardised diagnostic assessment tools which are guided by the DSM and ICD will be included in the review. It is accepted that there are limitations to this approach as coding of disorders can be often be applied without clinician interviews [58]. It is also important to note that there will be limitations to what diagnostic manual was used at the time of diagnosis as the diagnostic criteria would have changed over time for some of these disorders [58]. 
Furthermore, there are standardised diagnostic assessment tools which have been suggested to be male biased or thresholds of criteria too low to attain a diagnosis for varying reasons [59]. The review will follow other studies of similar methodology and include in the characteristics the description of the tools, codes and diagnostic manual used for transparency purposes. The review will acknowledge any limitations and address them in the review.

Table 1 Neurodevelopmental Disorders and Associated Synonyms

\begin{tabular}{|ll|}
\hline DISORDER & OTHER SYNONYMS \\
\hline Schizophrenia & \\
\hline Social phobia, unspecified & Paediatric bipolar disorder \\
\hline Obsessive compulsive disorder & Social anxiety or social anxiety phobia \\
\hline Eating disorders, unspecified & OCD \\
\hline Mild intellectual disability & Bulimia or anorexia \\
\hline $\begin{array}{l}\text { Social pragmatic communication } \\
\text { disorder }\end{array}$ & Developmental academic disorder or \\
\hline $\begin{array}{l}\text { Developmental disorder of speech } \\
\text { and language, unspecified }\end{array}$ & Searning difficulties \\
\hline $\begin{array}{l}\text { Specific reading disorder } \\
\text { Mathematics disorder }\end{array}$ & Reading disorder or written Disorder or Dyslexia \\
\hline $\begin{array}{l}\text { Specific developmental disorder of } \\
\text { motor function }\end{array}$ & Alcaculia or mathematic disability or Dyscalculia \\
\hline Autistic disorders & Developmental coordination disorder or Dyspraxia \\
\hline $\begin{array}{l}\text { Attention Deficit Hyperactivity } \\
\text { disorder }\end{array}$ & $\begin{array}{l}\text { Autism spectrum disorder, pervasive development disorder, } \\
\text { Reactive attachment disorder }\end{array}$ \\
\hline Tic disorder & RAD and (including Aspergers syndrome) \\
\hline Stereotyped mover \\
\hline
\end{tabular}

Stereotyped movement disorder

Foetal alcohol syndrome Foetal alcohol spectrum disorder or FASD

There will be no predefined health and social impacts or outcomes; due to the anticipated, limited studies in this area with regards to these vulnerable children and the specific associated disorders being explored. 
Predefining these might prohibit or exclude any important impacts or outcomes that might be affecting these children as a result of having these disorders.

\section{Study Selection}

The review will be undertaken by three reviewers. Two reviewers will critically analyse, code and appraise the selected studies. The third reviewer will be assigned as mediator; should the review need to reach consensus on any final selected studies.

Initially, all studies will be screened by review of title, followed by review of abstract as demonstrated by the search strategy already conducted (see Appendix 1).

The next stage will be to apply the exclusion and inclusion criteria and remove studies that do not fit the specified criterion. Following this, all remaining papers will be fully screened. As the study is exploring two areas of interest; the study selection will be divided into two stages. The first stage will select and appraise the selected studies that meet the inclusion criteria for the prevalence of these disorders and the second stage will appraise the selected studies that meet the inclusion criteria for impacts on health and social wellbeing.

The review will also ensure that the studies captured by the systematic reviews are not duplicated and that no significant or new literature has been missed during, preceding or proceeding when the systematic reviews were conducted. Intermittent searches will also be conducted while the review is being undertaken to ensure that new literature is not missed [60].

After the searches are conducted; a reviewer's meeting will be scheduled to seek consensus and to agree if any more primary studies should be included in the review; to aid in addressing the research questions.

\section{Data collection and extraction}

An extraction form will be designed to collate the information related to the areas of interest. Characteristics such as study name, country, total sample size, age, gender (\% male), type of placement, case ascertainment method, disorder, diagnostic system used, diagnostic instrument, number of cases of neurodevelopmental disorders and any impacts on health and social wellbeing. The form will systematically provide clear and unambiguous results to enable further analysis to occur [60].

\section{Quality and Bias assessment}

To screen and assess the quality of the literature in this review; the Joanna Briggs Institute (JBI) critical appraisal tools will be utilised [61]. These are appropriate tools that have been used in other reviews and suggested to be applicable due to the diversity of the designs normally anticipated in a systematic review [61]. All appraisal tools will address the bias in design, conduct and analysis [61]. For consistency purposes, two reviewers will independently assess and appraise the studies [60]. If there is a divergence in assessment, a review meeting will be arranged and the third reviewer will become mediator to reach 
consensus. This will be documented within the review as a narrative summary, to provide clarity and transparency.

\section{Data Synthesis and Analysis}

Data synthesis and analysis will be guided by the studies that are selected for review. If feasible. a metaanalysis will be conducted. The first stage will aim to estimate a pooled prevalence of the specific disorders in the LAC population versus the NoN-LAC population [60]. If sample sizes are too small the review will also transform the data into number of cases per population to enable an effect size to be calculated. [60].

It is anticipated that there may be high heterogeneity between studies, therefore, the random effect model will be used to estimate a mean of a distribution of effects [60,62]. Using this model should provide a more balanced weight to smaller and larger studies to estimate a more standardised mean effect. [60, 62]. Effect will be expressed as odds ratio with a proportion of $95 \%$ confidence intervals around the summary estimates. Forest plots will be used to provide a graphical representation of the results. To address publication bias, the funnel plot or trim and fill method will be used; although it is anticipated that the trim and fill method will be more appropriate due to the smaller studies associated with the care experienced population [60, 62-63].

Subsequently, where statistical analysis is not possible; a descriptive analysis will be provided detailing the prevalence ranges of the neurodevelopmental disorders (eg, ADHD ranged from $2 \%$ to $16 \%$ in six of the studies) and characteristics of the studies.

A thematic analysis will be used to enable a framework and narrative synthesis to occur. All information relating to the impacts on the health and social wellbeing of this population; as a result of having these neurodevelopmental disorders will be double coded by two reviewers. In the final stage, all analyses will be amalgamated to provide a discussion of the results attained.

\section{Software considerations}

The SUMARI software package, a comprehensive review management system that has been designed to assist researchers in the health and social sciences to conduct and support systematic reviews will be utilised to extract, critically appraise and part analyse the data [64]. To conduct the meta-analysis; the RevMan 5.0 software package will be used to meet the needs of the research design [65].

\section{Dissemination and Research Integrity}

The findings will be disseminated through various pathways, such as peer review journals, public and third sector organisations, Welsh government policy departments, the Children's Commissioner, appropriate paediatric National and International Conferences; using various methods such as posters, websites and presentations. 


\section{Limitations}

The protocol anticipates that there will be several limitations to acknowledge, due to the complexities that surround this population. One limitation may be that there will be differences in the definition of a LAC or NoN-LAC; which will be noted and addressed in the review should it impact the results in anyway. It is also acknowledged that diagnostic criteria will have evolved and changed; in relation to the classification of these disorders outlined in the previous and current ICD and DSM. Although, the rationale for making the upper age limit 25 is detailed, a limitation to this could be that the review excludes older individuals with or without these disorders and possibly additional information regarding health and social wellbeing. Any limitations that arise during the review process will be documented and included within the final systematic review publication.

\section{Discussion}

Early identification and early intervention are key components to improving health and social wellbeing. Rates of children entering the care system are increasing on a global level; demands on services are placing immense pressures on government funding, third sectors, front line services, families and more importantly, on the children themselves $[66,67]$. With data collection and diagnostic accuracy progressing at a considerable rate; this review will contribute to the exploration and prevalence of these neurodevelopmental disorders, that are often and sometimes very difficult to diagnose. For this vulnerable, high-risk population who are embedded in services, early identification and intervention are still unattainable for many, due to the multi-faceted complexities that encompass their lives. Therefore, from an impact perspective, the results of this review could provide policy and services with key information that might further explain what dynamics may be driving demands into children service and aid in directing appropriate services to meet the needs of these children.

\section{Abbreviations}

LAC: Looked after child/children, ICD: International Classification of Diseases, DSM: Diagnostic and Statistical Manual of Mental Disorders.

\section{Declarations}

\section{Ethics approval and consent to participate}

Not applicable. Due to the nature of this review, no ethical approval is needed. However, ethical considerations will be acknowledged throughout the review; if deemed appropriate [41].

\section{Consent for publication}

Not applicable. 
Availability of data and materials

Not applicable.

\section{Competing interests}

The authors declare there are no competing interests.

\section{Funding}

Co-funded by the Health and Care Research Wales and the Economic Social Research Council (ESRC) Wales.

\section{Authors' contributions}

All authors detailed on the title page will be involved in the analysis and interpretation of results. All authors have been involved in the design of the study and will revise and approve the final manuscript.

\section{Acknowledgements}

None

\section{References}

1. Kääriälä A, Hiilamo H. Children in out-of-home care as young adults: A systematic review of outcomes in the Nordic countries. Children and Youth services review. 2017 Aug 1;79:107-14.

2. O'Higgins A, Sebba J, Luke N. What is the relationship between being in care and the educational outcomes of children. An international systematic review. 2015 Sep.

3. Sebba J, Berridge D, Luke N, Fletcher J, Bell K, Strand S, Thomas S, Sinclair I, O'Higgins A. The educational progress of looked after children in England: Linking care and educational data. University of Oxford Department of Education/University of Bristol; 2015 Jan 1.

4. Mezey G, Robinson F, Gillard S, Mantovani N, Meyer D, White S, Bonell C. Tackling the problem of teenage pregnancy in looked-after children: a peer mentoring approach. Child \& Family Social Work. 2017 Feb;22(1):527-36.

5. Lehmann S, Havik OE, Havik T, Heiervang ER. Mental disorders in foster children: a study of prevalence, comorbidity and risk factors. Child and adolescent psychiatry and mental health. 2013 Dec;7(1):39.

6. Evans R, White J, Turley R, Slater T, Morgan H, Strange H, Scourfield J. Comparison of suicidal ideation, suicide attempt and suicide in children and young people in care and non-care populations: Systematic review and meta-analysis of prevalence. Children and Youth Services Review. 2017 Nov 1;82:122-9. 
7. Fitzpatrick C, Williams P, Coyne D. Supporting looked after children and care leavers in the criminal justice system: emergent themes and strategies for change. Prison Service Journal. 2016 Jul 5(226):8-13.

8. Vinnerljung B, Sallnäs M. Into adulthood: a follow-up study of 718 young people who were placed in out-of-home care during their teens. Child \& Family Social Work. 2008 May;13(2):144-55.

9. Welsh Assembly Government (WAG) The Parliamentary Review of Health and Social Care in Wales (2018). Accessed 29th October 2019 at: http://senedd.assembly.wales/mglssueHistoryHome.aspx? Ild=17296\&Opt=0

10. Children Act 1989 Accessed 25 March 2019 at: http://www.legislation.gov.uk/ukpga/1989/41/section/2

11. Sanders J, Munford R, Liebenberg L, Ungar M. Multiple service use: The impact of consistency in service quality for vulnerable youth. Child abuse \& neglect. $2014 \mathrm{Apr}$ 1;38(4):687-97.

12. Morris L, Salkovskis P, Adams J, Lister A, Meiser-Stedman R. Screening for post-traumatic stress symptoms in looked after children. Journal of Children's Services. 2015 Dec 21;10(4):365-75.

13. Häggman-Laitila A, Salokekkilä P, Karki S. Transition to adult life of young people leaving foster care: A qualitative systematic review. Children and Youth Services Review. 2018 Dec 1;95:134-43.

14. Gilbert R, Widom CS, Browne K, Fergusson D, Webb E, Janson S. Burden and consequences of child maltreatment in high-income countries. The lancet. 2009 Jan 3;373(9657):68-81.

15. Brown A, Waters CS, Shelton KH. A systematic review of the school performance and behavioural and emotional adjustments of children adopted from care. Adoption \& Fostering. 2017 Dec;41(4):346-68.

16. Einfeld SL, Ellis LA, Emerson E. Comorbidity of intellectual disability and mental disorder in children and adolescents: A systematic review. Journal of Intellectual and Developmental Disability. 2011 Jun $1 ; 36(2): 137-43$.

17. Department for Education (DfE) 2017. Guidance on Looked After Children with Special Educational Needs placed out-of-authority. Accessed 3rd Match 2019 at: https://dera.ioe.ac.uk/662/1/000602010DOM-EN.pdf

18. NHS (2018) 'Mental Health of Children and Young People in England, 2017 [PAS]' Accessed 3rd March at: https://digital.nhs.uk/data-and-information/publications/statistical/mental-health-ofchildren-and-young-people-in-england/2017/2017\#key-facts

19. NSPCC (2019) Statistics briefing: looked after children. Accessed 3rd March, 2019 at: https://learning.nspcc.org.uk/media/1622/statistics-briefing-looked-after-children.pdf

20. Ford T, Vostanis P, Meltzer H, Goodman R. Psychiatric disorder among British children looked after by local authorities: comparison with children living in private households. The British Journal of Psychiatry. 2007 Apr;190(4):319-25.

21. Meltzer H, Gatward R, Corbin T, Goodman R, Ford T. The mental health of young people looked after by local authorities in England. London: The Stationery Office. 2003. 
22. Lightfoot E, Hill K, LaLiberte T. Prevalence of children with disabilities in the child welfare system and out of home placement: An examination of administrative records. Children and Youth Services Review. 2011 Nov 1;33(11):2069-75.

23. Hill L, Baker C, Kelly B, Dowling S. Being counted? Examining the prevalence of looked-after disabled children and young people across the UK. Child \& Family Social Work. 2017 Feb;22(1):287-95.

24. Palmier-Claus JE, Berry K, Bucci S, Mansell W, Varese F. Relationship between childhood adversity and bipolar affective disorder: systematic review and meta-analysis. The British Journal of Psychiatry. 2016 Dec;209(6):454-9.

25. Bethell C, Gombojav N, Solloway M, Wissow L. Adverse childhood experiences, resilience and mindfulness-based approaches: common denominator issues for children with emotional, mental, or behavioral problems. Child and Adolescent Psychiatric Clinics. 2016 Apr 1;25(2):139-56.

26. Saarni SI, Suvisaari J, Sintonen H, Pirkola S, Koskinen S, Aromaa A, Lönnqvist J. Impact of psychiatric disorders on health-related quality of life: general population survey. The British journal of psychiatry. 2007 Apr;190(4):326-32.

27. Masson M, East-Richard C, Cellard C. A meta-analysis on the impact of psychiatric disorders and maltreatment on cognition. Neuropsychology. 2016 Feb;30(2):143.

28. D'Souza H, Karmiloff-Smith A. Neurodevelopmental disorders. Wiley Interdisciplinary Reviews: Cognitive Science. 2017 Jan;8(1-2):e1398.

29. Schork AJ, Won H, Appadurai V, Nudel R, Gandal M, Delaneau O, Hougaard D, Baekved-Hansen M, Bybjerg-Grauholm J, Pedersen MG, Pedersen CB. A genome-wide association study for shared risk across major psychiatric disorders in a nation-wide birth cohort implicates fetal neurodevelopment as a key mediator. bioRxiv. 2017 Jan 1:240911.

30. Lo MT, Hinds DA, Tung JY, Franz C, Fan CC, Wang Y, Smeland OB, Schork A, Holland D, Kauppi K, Sanyal N. Genome-wide analyses for personality traits identify six genomic loci and show correlations with psychiatric disorders. Nature genetics. 2017 Jan;49(1):152.

31. Thapar A, Cooper M, Rutter M. Neurodevelopmental disorders. The Lancet Psychiatry. 2017 Apr $1 ; 4(4): 339-46$.

32. Young S, González RA, Mullens H, Mutch L, Malet-Lambert I, Gudjonsson GH. Neurodevelopmental disorders in prison inmates: comorbidity and combined associations with psychiatric symptoms and behavioural disturbance. Psychiatry research. 2018 Mar 1;261:109-15.

33. Wolff JJ, Hazlett HC, Lightbody AA, Reiss AL, Piven J. Repetitive and self-injurious behaviors: associations with caudate volume in autism and fragile $X$ syndrome. Journal of neurodevelopmental disorders. 2013 Dec;5(1):12.

34. Dimitropoulos A, Ho A, Feldman B. Social responsiveness and competence in Prader-Willi syndrome: Direct comparison to autism spectrum disorder. Journal of autism and developmental disorders. 2013 Jan 1;43(1):103-13.

35. Guthrie W, Swineford LB, Nottke C, Wetherby AM. Early diagnosis of autism spectrum disorder: stability and change in clinical diagnosis and symptom presentation. Journal of Child Psychology 
and Psychiatry. 2013 May;54(5):582-90.

36. Madsen KB, Ravn MH, Arnfred J, Olsen J, Rask CU, Obel C. Characteristics of undiagnosed children with parent-reported ADHD behaviour. European child \& adolescent psychiatry. 2018 Feb 1;27(2):14958.

37. Tierney S, Burns J, Kilbey E. Looking behind the mask: Social coping strategies of girls on the autistic spectrum. Research in Autism Spectrum Disorders. 2016 Mar 1;23:73-83.

38. Bargiela S, Steward R, Mandy W. The experiences of late-diagnosed women with autism spectrum conditions: An investigation of the female autism phenotype. Journal of Autism and Developmental Disorders. 2016 Oct 1;46(10):3281-94.

39. Salim E, Fleming M, MacKay DF, Henderson A, Kinnear D, Clark D, King A, McLay JS, Cooper SA, Pell JP. Neurodevelopmental multimorbidity and educational outcomes of 766,244 Scottish schoolchildren: Michael Fleming. European Journal of Public Health. 2019 Nov 1;29(Supplement_4):ckz185-164.

40. Barneveld PS, Swaab H, Fagel S, van Engeland H, de Sonneville LM. Quality of life: A case-controlled long-term follow-up study, comparing young high-functioning adults with autism spectrum disorders with adults with other psychiatric disorders diagnosed in childhood. Comprehensive psychiatry. 2014 Feb 1;55(2):302-10.

41. ICD 10 (2018) ICD 10 Data 'Mental, Behavioural and Neurodevelopmental disorders F01-F99'. Accessed 3rd March 2019 at: https://www.icd10data.com/ICD10CM/Codes/F01-F99

42. Willis R, Dhakras S, Cortese S. Attention-deficit/hyperactivity disorder in looked-after children: A systematic review of the literature. Current developmental disorders reports. 2017 Sep 1;4(3):78-84.

43. Ferguson CJ, Brannick MT. Publication bias in psychological science: prevalence, methods for identifying and controlling, and implications for the use of meta-analyses. Psychological methods. 2012 Mar; 17(1):120.

44. Zwaigenbaum L, Bryson S, Garon N. Early identification of autism spectrum disorders. Behavioural brain research. 2013 Aug 15;251:133-46.

45. Sauver JL, Barbaresi WJ, Katusic SK, Colligan RC, Weaver AL, Jacobsen SJ. Early life risk factors for attention-deficit/hyperactivity disorder: a population-based cohort study. InMayo Clinic Proceedings 2004 Sep 1 (Vol. 79, No. 9, pp. 1124-1131). Elsevier.

46. Pritchett R, Hockaday H, Anderson B, Davidson C, Gillberg C, Minnis H. Challenges of assessing maltreated children coming into foster care. The Scientific World Journal. 2016;2016.

47. Chasnoff IJ, Wells AM, King L. Misdiagnosis and missed diagnoses in foster and adopted children with prenatal alcohol exposure. Pediatrics. 2015 Feb 1;135(2):264-70.

48. Pritchett R, Hockaday H, Anderson B, Davidson C, Gillberg C, Minnis H. Challenges of assessing maltreated children coming into foster care. The Scientific World Journal. 2016;2016.

49. Smeland OB, Bahrami S, Frei O, Shadrin A, O'Connell K, Savage J, Watanabe K, Krull F, Bettella F, Steen NE, Ueland T. Genome-wide analysis reveals extensive genetic overlap between schizophrenia, bipolar disorder, and intelligence. Molecular psychiatry. 2019 Jan 4:1. 
50. Moher D, Shamseer L, Clarke M, Ghersi D, Liberati A, Petticrew M, Shekelle P, Stewart LA. Preferred reporting items for systematic review and meta-analysis protocols (PRISMA-P) 2015 statement. Systematic reviews. 2015 Dec;4(1):1.

51. Gough D, Thomas J, Oliver S. Clarifying differences between review designs and methods. Systematic reviews. 2012 Dec;1(1):28.

52. Jahan N, Naveed S, Zeshan M, Tahir MA. How to conduct a systematic review: a narrative literature review. Cureus. 2016 Nov;8(11).

53. Gough D, Richardson M. Systematic reviews. In Advanced Research Methods for Applied Psychology 2018 Aug 14 (pp. 75-87). Routledge.

54. Xu Y, Bright CL. Children's mental health and its predictors in kinship and non-kinship foster care: A systematic review. Children and Youth Services Review. 2018 Jun 1;89:243-62.

55. Bronsard G, Alessandrini M, Fond G, Loundou A, Auquier P, Tordjman S, Boyer L. The prevalence of mental disorders among children and adolescents in the child welfare system: A systematic review and meta-analysis. Medicine. 2016 Feb;95(7).

56. Jedwab M, Shaw TV. Predictors of reentry into the foster care system: Comparison of children with and without previous removal experience. Children and Youth Services Review. 2017 Nov 1;82:17784.

57. First MB, Reed GM, Hyman SE, Saxena S. The development of the ICD-11 clinical descriptions and diagnostic guidelines for mental and behavioural disorders. World Psychiatry. 2015 Feb;14(1):82-90.

58. Merten EC, Cwik JC, Margraf J, Schneider S. Overdiagnosis of mental disorders in children and adolescents (in developed countries). Child and adolescent psychiatry and mental health. 2017 Dec $1 ; 11(1): 5$.

59. Haney JL. Autism, females, and the DSM-5: Gender bias in autism diagnosis. Social Work in Mental Health. 2016 Jul 3;14(4):396-407.

60. Bashir Y, Conlon KC. Step by step guide to do a systematic review and meta-analysis for medical professionals. Irish Journal of Medical Science (1971-). 2018 May 1;187(2):447-52.

61. Munn Z, Moola S, Riitano D, Lisy K. The development of a critical appraisal tool for use in systematic reviews addressing questions of prevalence. International journal of health policy and management. 2014 Aug;3(3):123.

62. Ward MM. Estimating disease prevalence and incidence using administrative data: some assembly required.

63. Duval S, Tweedie R. A nonparametric "trim and fill" method of accounting for publication bias in meta-analysis. Journal of the American Statistical Association. 2000 Mar 1;95(449):89-98.

64. Munn Z. Software to support the systematic review process: the Joanna Briggs Institute System for the Unified Management, Assessment and Review of Information (JBI-SUMARI).

65. Zeng W, Chen R, Wang X, Zhang Q, Deng W. Prevalence of mental health problems among medical students in China: A meta-analysis. Medicine. 2019 May;98(18). 
66. Sanmartin MX, Ali MM, Lynch S. Foster care admissions and state-level criminal justice-focused prenatal substance use policies. Children and Youth Services Review. 2019 Jul 1;102:102-7.

67. Crea TM, Lopez A, Taylor T, Underwood D. Unaccompanied migrant children in the United States: Predictors of placement stability in long term foster care. Children and Youth Services Review. 2017 Feb 1;73:93-9. 